\title{
Determination of Deformation Characteristics of Bionic Periodic Isolation Unit for Ship Engine from Vibration of Sensor
}

\author{
Liao Jianbin, ${ }^{1,2,3}$ Yu Hong Liang, ${ }^{2,3 *}$ Song Yuchao, ${ }^{1}$ and Guo Xueping ${ }^{2}$ \\ ${ }^{1}$ Marine Engineering College, Dalian Maritime University, Dalian 116026, China \\ ${ }^{2}$ Marine Engineering College, Jimei University, Xiamen, Fujian 361021, China \\ ${ }^{3}$ Fujian Province Key Laboratory of Ship and Ocean Engineering, Xiamen, Fujian 361021, China
}

(Received May 5, 2019; accepted September 10, 2019)

Keywords: bionic organ structure, periodic cell, static load, stress

On the basis of the composition of organs and static theory, a bionic periodic isolation structure is modeled to study the mechanical characteristics of vibration isolation with the aim of isolating ship vibration. The unit cell of the structure is composed of three layers, an upper steel layer, a middle stiff rubber layer, and a bottom soft rubber layer, and some multicell units are analyzed. To maintain numerical accuracy, the mapping method is used to mesh the periodic model with an element size of $0.01 \mathrm{~mm}$. In the case of a static load, all the unit cells exhibit bulging deformation on two sides. The maximum deformation is not always at the upper layer, with the maximum bulging at two sides in the single-cell case and deformation in the middle layer in the cases of 9 and 10 cells. When the number of cells is above 6 , deformation is enhanced in the middle layer, with half a sinusoidal wave. In this paper, the deformation ratio in the middle layer is as high as $87.99 \%$. In addition, the stress distribution characteristic is also compared among some cases. The bionic isolation structure and its analysis may provide a new way to reduce the vibration in a ship.

\section{Introduction}

In recent years, with the development of the internal combustion engine leading to high speed and power, the diesel engine has become the main source of ship vibration and noise. Vibration affects the performance of instruments and equipment, reduces their reliability, and causes additional power loss. The vibration and noise generated by the operation of equipment are not only related to the normal operation of equipment and the comfort of living conditions on board, but also have a great impact on the industrial land environment. Therefore, a large amount of research on vibration, including that on vibration isolation theory and techniques, has been published. ${ }^{(1-6)}$

Bionic technology and its theoretical research have promoted the integration and promotion of multidisciplinary technology theory. The concise, complex, and diversified structures and profiles formed by the evolution of species over billions of years provide unlimited design resources. Bionics is an interdisciplinary subject based on life science, mathematics, mechanics,

*Corresponding author: e-mail: yu1202@hotmail.com

https://doi.org/10.18494/SAM.2019.2505 
and engineering technology. For example, the invention of fishing nets may have originated from the imitation of spider webs in ancients times, the airfoil of an airplane imitates the profile of a bird's wing, the jet propulsion principle imitates the motion principle of cuttlefish, and the invention of radar originates from the imitation of a bat's ultrasonic positioning. A bionic periodic structure can be used to sense deformation in industrial applications, ${ }^{(7)}$ especially when it is used as a sensor in a vibration monitoring system. The characteristic deformation of tissues can present a novel investigation in sensing element development.

Innovative studies on vibration isolation based on bionic theory, including bionic muscle, and the suppression of organs by vibration are very meaningful. ${ }^{(7-12)}$ In this study, an organ is modeled by a cell consisting of three layers, and the effect of the number of periodic cell units is studied to analyze the deformation of the structure.

\section{Structure of Bionic Model}

As shown in Fig. 1, an animal organ is composed of the epidermis, dermis, and subcutaneous tissue. In this study, on the basis of bionic theory, the cell is composed of three layers: the upper layer is structural steel with height $h_{1}$, the middle layer is stiff rubber with height $h_{2}$, and the lower layer is soft rubber with height $h_{3}$. In this work, the length of the cell is $20 \mathrm{~mm}, h_{1}$ is $4 \mathrm{~mm}, h_{2}$ is $6 \mathrm{~mm}$, and $h_{3}$ is $10 \mathrm{~mm}$. In the analysis, cases with different numbers of cells are studied (Fig. 2).

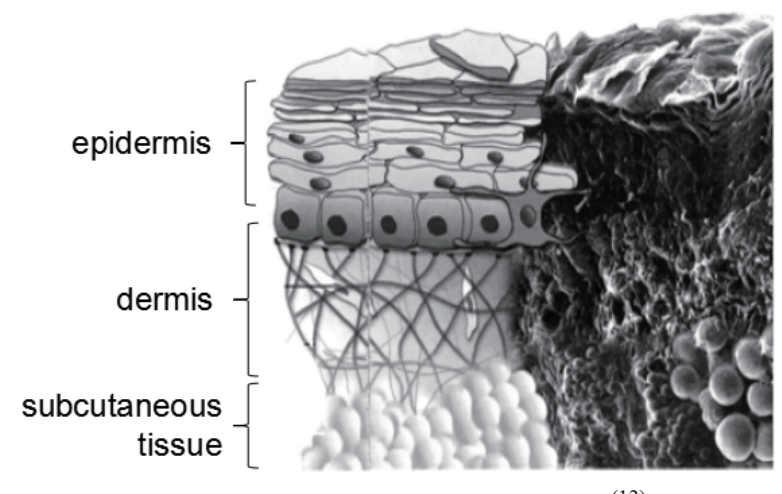

Fig. 1. Schematic of animal organ. ${ }^{(13)}$

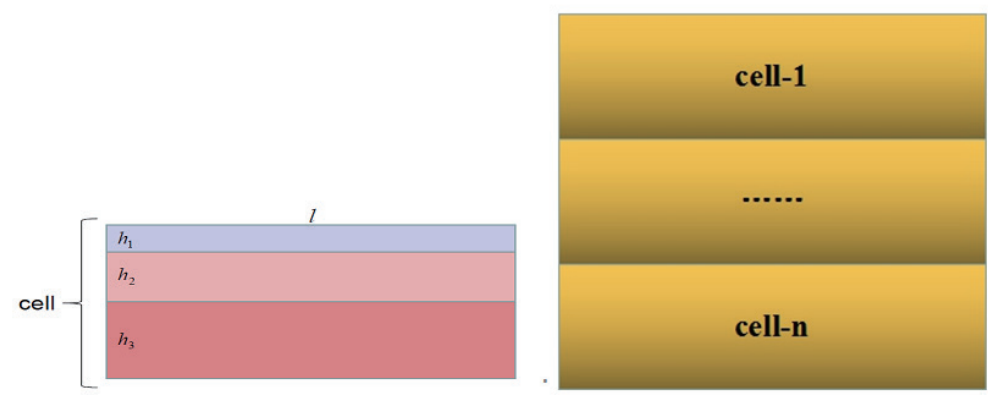

Fig. 2. (Color online) Schematic of analysis model. 


\section{Load Formula Used in Analysis}

For a common two-dimensional (2D) mass-spring system, the static force equation is

$$
F=k x
$$

Considering the system deformation of the rubber in the system, the in-plane steady-state deformation should be expressed as

$$
-\nabla \cdot \sigma=F_{V},
$$

where $\nabla$ is the Laplace operator, $\sigma$ is the stress tensor, and $F_{V}$ is the load.

The linear elastic material of structural steel in the model satisfies

$$
\{\sigma\}=[D]\{\varepsilon\}
$$

where $\{\sigma\}$ is the stress matrix, $[D]$ is the elasticity matrix, and $\{\varepsilon\}$ is the strain matrix.

For the hyperelastic material of rubber, the Mooney-Rivlin model ${ }^{(14)}$ is

$$
W=C_{10}\left(I_{1}-3\right)+C_{01}\left(I_{2}-3\right)
$$

where $W$ is the strain energy density, $C_{10}$ and $C_{01}$ are the material parameters, and $I$ is the Rivlin strain hypothesis.

\section{Numerical Analysis}

The upper layer is modeled by structural steel with a density of $7850 \mathrm{~kg} / \mathrm{m}^{3}$, $E=2.1 \times 10^{11} \mathrm{~Pa}$, and $\mu=0.33$. The Mooney-Rivlin two-parameter model with $C_{10}=0.7691 \times$ $10^{6}, C_{01}=0.0199 \times 10^{6}$, and $p=1100 \mathrm{~kg} / \mathrm{m}^{3}$ is used for the middle layer of stiff rubber, and the same model is used for the lower layer of soft rubber but with $C_{10}=0.1950 \times 10^{6}$ and $C_{01}=0.0162$ $\times 10^{6}$. The mapped mesh method is used to build the definition domain, as shown in Fig. 3 . The maximum element size is $0.4 \mathrm{~mm}$, the minimum element size is $0.01 \mathrm{~mm}$, and the element quality is 1 .

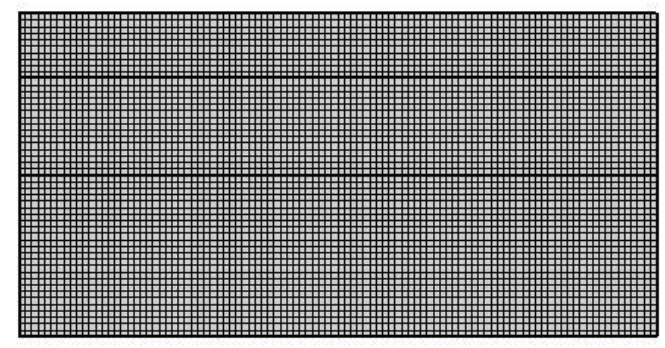

Fig. 3. Mesh used in single-cell model. 
During analysis, the static load is below $100 \mathrm{~N}$ in the positive $Y$ direction at the midpoint of the upper surface. The bottom line has a fixed boundary constraint. In the static analysis, the deformation is the main parameter, so the displacement distributions of the whole structure, the middle line, and the midpoint of the upper surface are analyzed to study the deformation characteristics of bionic periodic structures.

Ten cases of deformation of the whole structure are shown in Fig. 4, and the deformation is symmetrical. For one cell, the maximum deformation is on the two sides of the lower soft rubber, with swelling of $0.01 \mathrm{~mm}$, but the upper layer only has a $0.005 \mathrm{~mm}$ displacement. A large amount of strain energy is absorbed by the side deformation, so the vertical deformation is small. With increasing number of cells, the location of maximum deformation changes to the top cell, in which the lower soft layer still has the maximum displacement. This phenomenon appears in the case of 2-8 cells, with a constant deformation of $0.01 \mathrm{~mm}$. In the case of 2-7 cells, the deformation distribution periodically increases from the bottom to the top. In case No. 8,

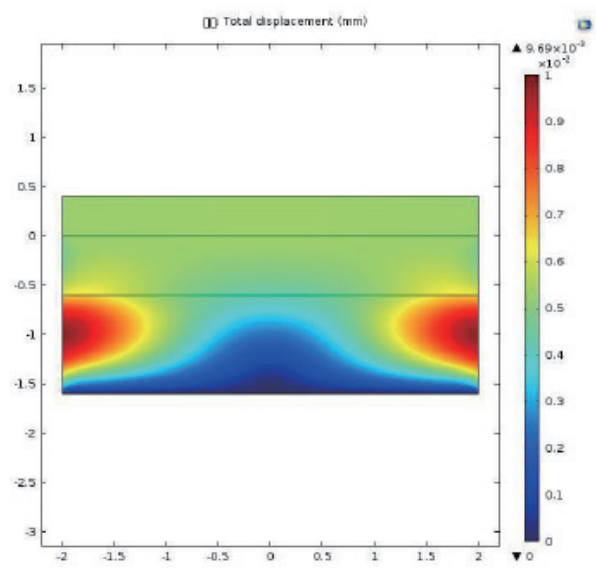

(a)

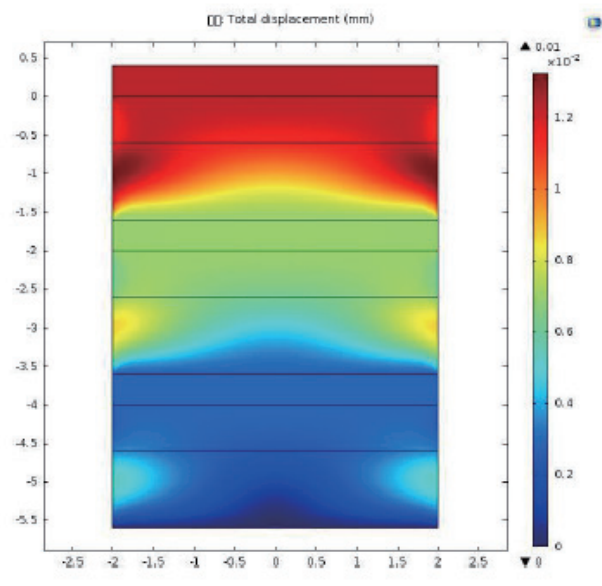

(c)

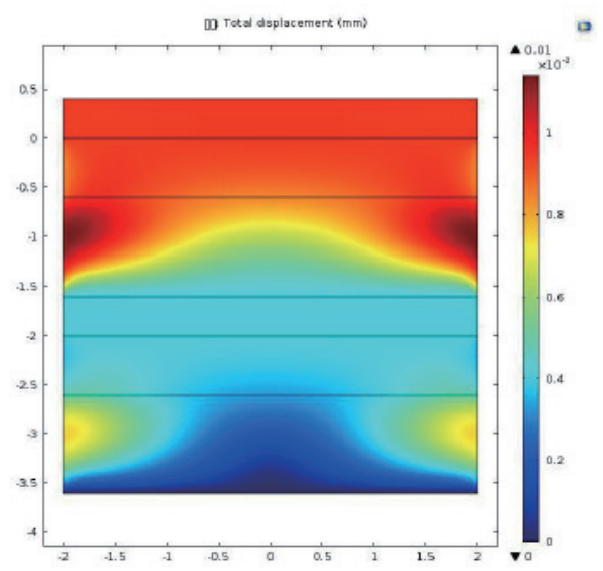

(b)

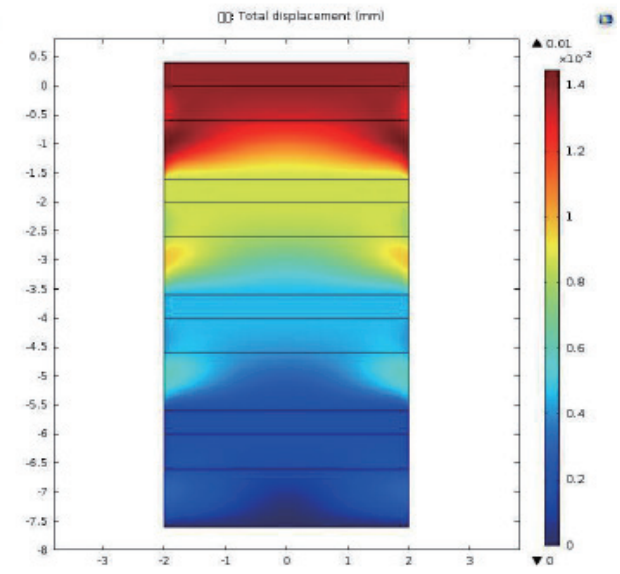

(d)

Fig. 4. (Color online) Displacement contours of isolator. (a)-(d) are Nos. 1-4, respectively. 


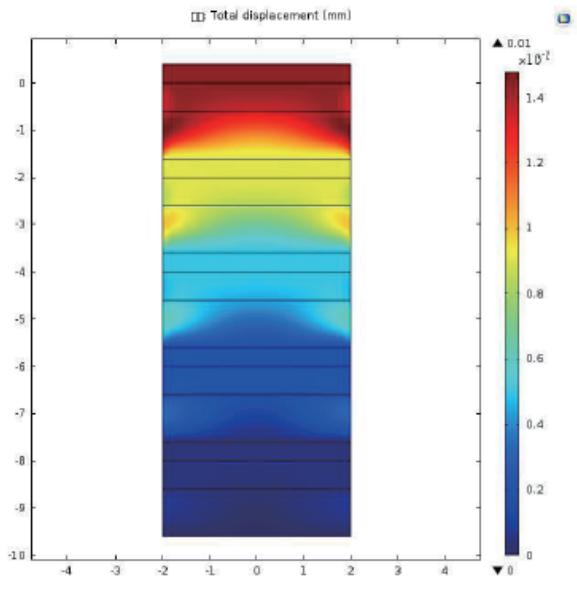

(e)

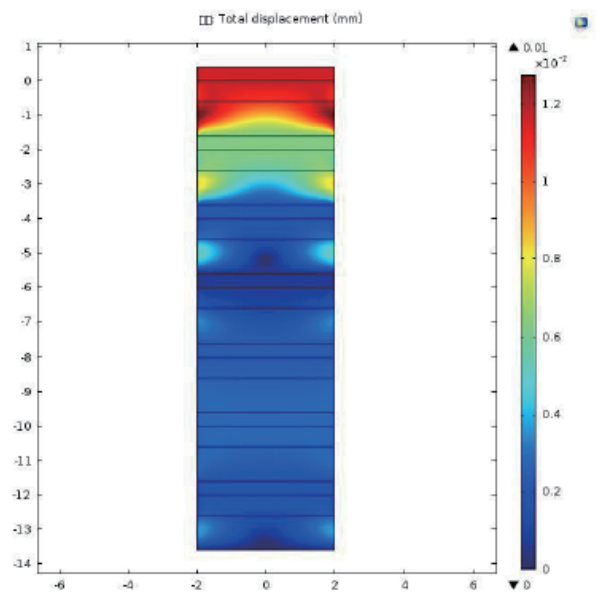

(g)

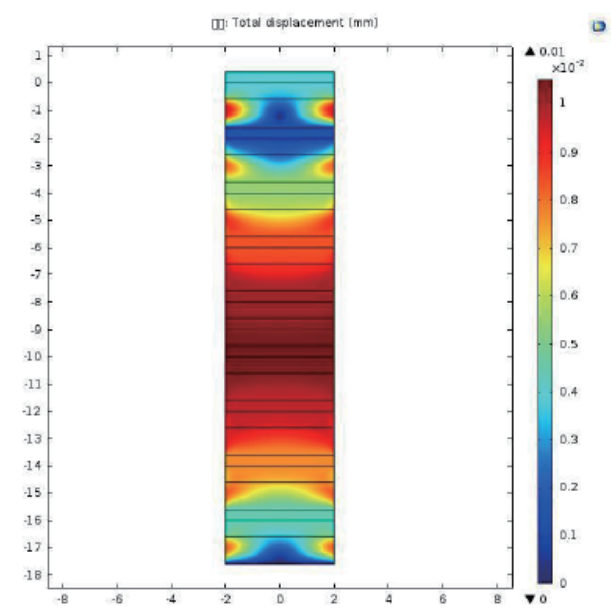

(i)

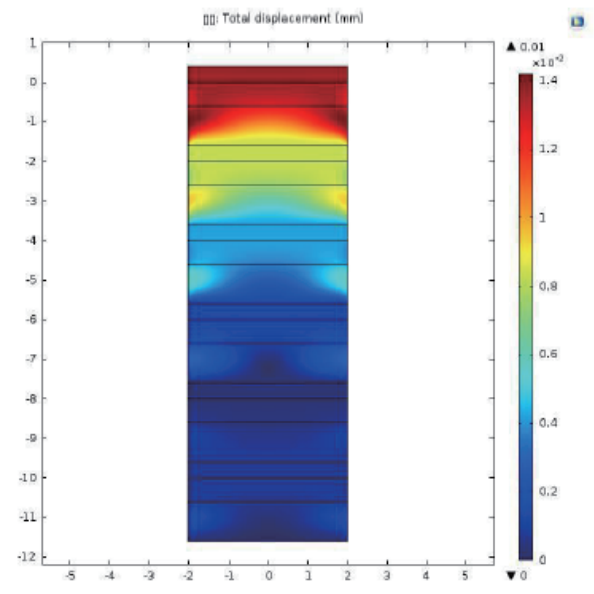

(f)

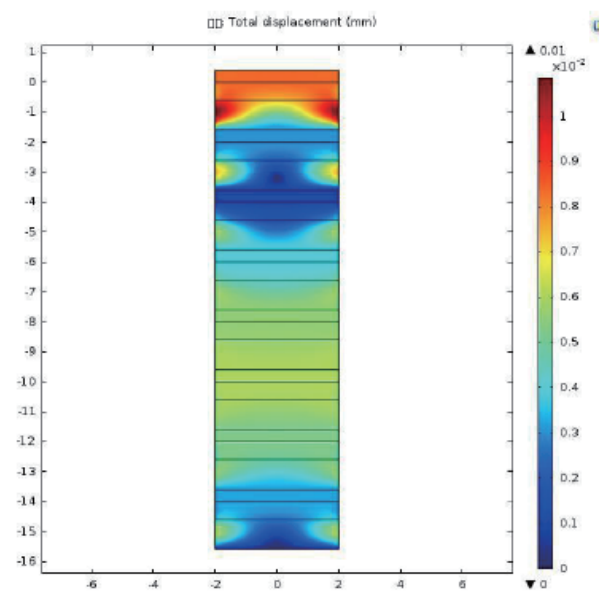

(h)

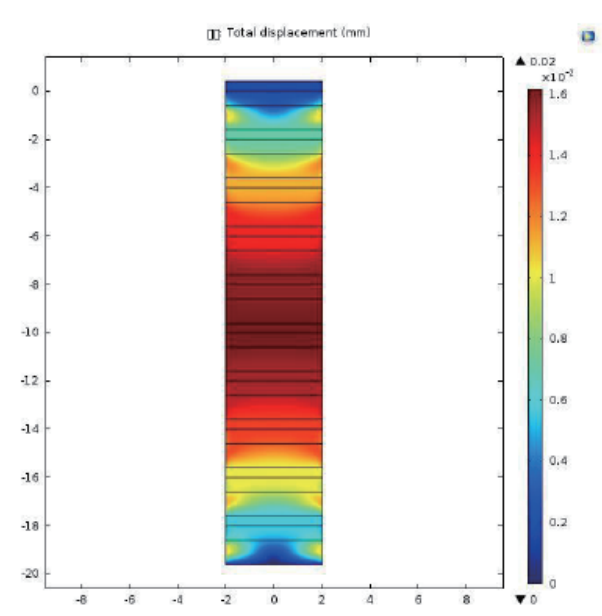

(j)

Fig. 4. (Color online) (Continued) Displacement contours of isolator. (e)-(j) are Nos. 5-10, respectively. 
there is a large deformation in the middle layer in the vertical direction. In cases No. 9 and No. 10 , the maximum deformation of $0.01 \mathrm{~mm}$ is in the middle, which is different from that in case No. 8. The displacements of the top layer are only 0.004 and $0.001 \mathrm{~mm}$ in cases No. 9 and No. 10, respectively. The variation in deformation can be as high as $90 \%$.

The deformation distributions of the middle line in the analyzed cases are shown in Fig. 5. In the case of 1-5 cells, the deformation gradually increases with the numbers of cells. The curves in this case have slopes at the layer intersections. In case No. 6, there is a sinusoidal wave in the bottom section. In case No. 10, the sinusoidal wave becomes a large arc, with the maximum deformation in the middle and the minimum deformation at the two ends. The displacement of the midpoint of the upper layer is shown in Table 1. Case No. 10 has a minimum deformation of $1.73 \times 10^{-3} \mathrm{~mm}$. The relative difference in deformation is as high as $87.99 \%$.

The stress concentration in many engineering structures can cause cracking of the structure; in this case, the stress distribution of the lowest cell is also derived by further analysis, where the height of the lowest cell is $20 \mathrm{~mm}$ in every case. The von Mises stresses of five of the cases are shown in Fig. 6. It is clear that the stress distribution has the same trend in all cases. At the fixed bottom with $h=0 \mathrm{~cm}$, the stress in case No. 1 is the largest and is as high as $7984.36 \mathrm{~N} / \mathrm{m}^{2}$; in cases No. 3, No. 5, No. 7, and No. 9, the stresses are reduced by 46.94, 94.17, 58.35 , and $10.61 \%$, respectively. Among the analyzed cases, case No. 5 has the minimum stress, and the peak stress is located near $h=1.6 \mathrm{~cm}$.

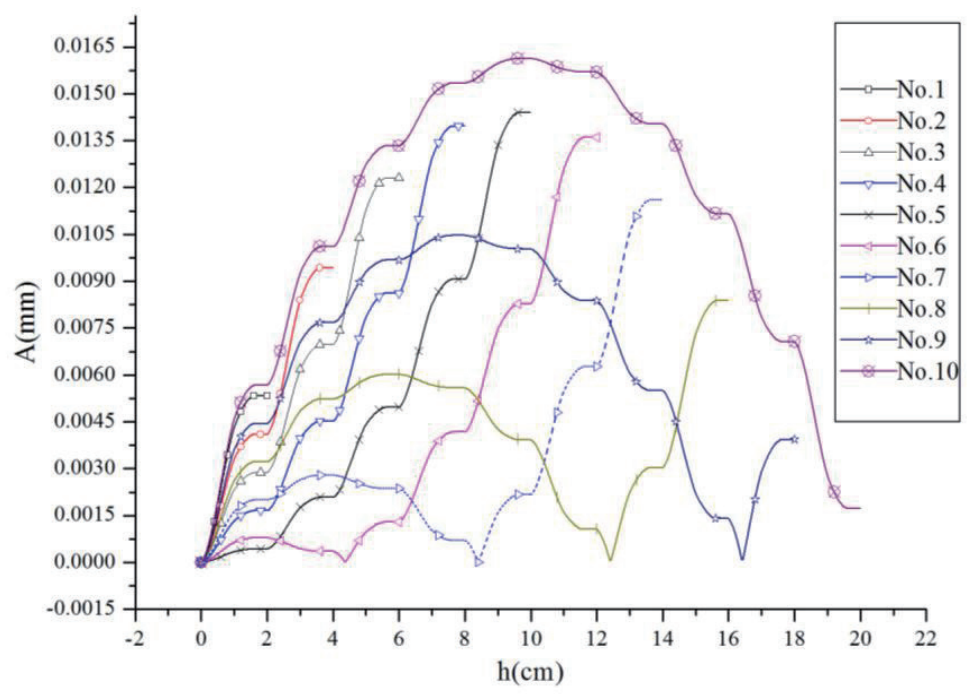

Fig. 5. (Color online) Displacement distributions of vertical middle line.

Table 1

Vertical displacement of midpoint of upper layer.

\begin{tabular}{ccccccccccc}
\hline & No. 1 & No. 2 & No. 3 & No. 4 & No. 5 & No. 6 & No. 7 & No. 8 & No. 9 & No. 10 \\
\hline$Y(0.001 \mathrm{~mm})$ & 5.34 & 9.44 & 12.31 & 13.96 & 14.40 & 13.61 & 11.60 & 8.38 & 3.93 & 1.73 \\
\hline
\end{tabular}




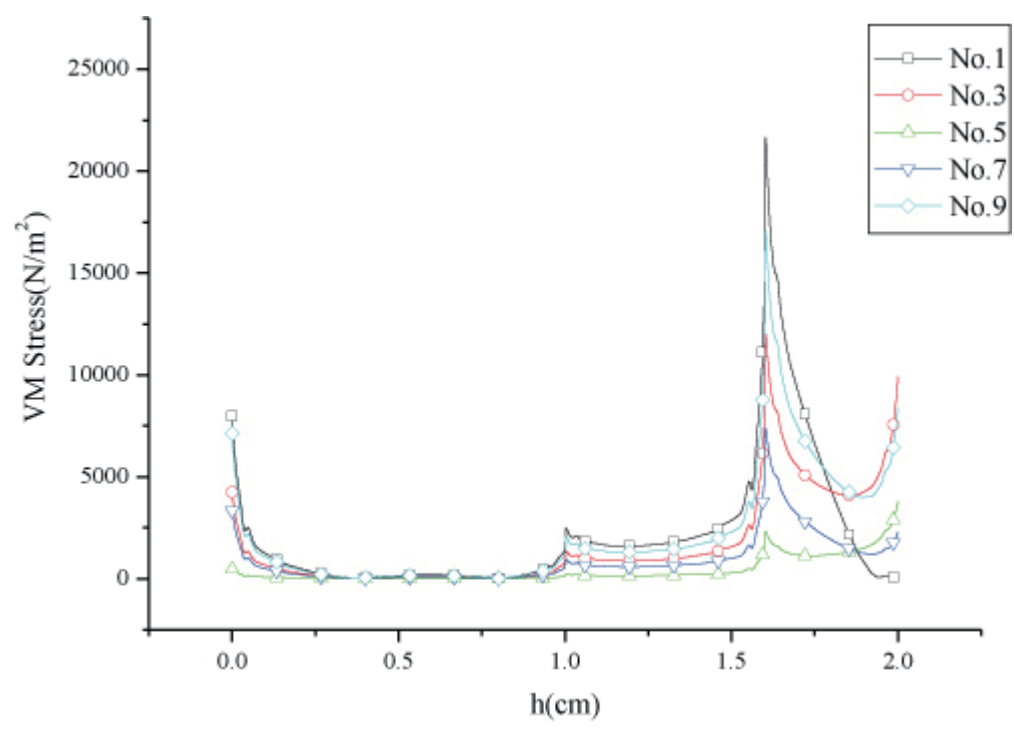

Fig. 6. (Color online) Von mises stress distributions.

\section{Conclusions}

Focusing on the vibration isolation problem, a bionic periodic structure was modeled on the basis of the organ structure. The results of static load analysis showed that the maximum deformation displacement was not always at the top layer surface owing to the side swelling of the rubber layer, which absorbs most of the strain energy. In this paper, the number of periodic cells ranged from 1 to 10. Case No. 1 had a small displacement of the top layer, and case No. 10 had the least deformation displacement, with a $90 \%$ decrease in the whole plane and a $87.99 \%$ decrease at the mid point. Regarding the stress, case No. 5 showed the minimum stress, $94.17 \%$ less than that of a single cell. The next step is to carry out transient analysis and tests to reveal the characteristics of the bionic periodic isolation structure.

\section{Acknowledgments}

This study and some of the analyses were carried out with the assistance of Fujian Province Key Laboratory of Ship and Ocean Engineering and Fujian Engineering Research Center of Marine Detecting and Remanufacturing. The authors gratefully acknowledge Fujian Science and Technology Projects (No. 2019H0020).

\section{References}

1 Z. Wei, B. Li, J. Du, L. Lin, and F. Tan: J Mech. Eng. 52 (2016) 91. https://doi.org/10.3901/JME.2016.15.091

2 Y. Chen, Q. Huang, and H. Shi: J. Low. Freq. Noise 25 (2005) 5. https://doi.org/10.3969/ j.issn.1006-1355.2005.05.001

3 J. Li and S. Li: J. Vib. Shock 37 (2018) 1. https://doi.org/10.13465/j.cnki.jvs.2018.01.025

4 Y. Wang, Z. Liu, Q. Huang, and M. Zhou: Int. J. Mech. Mater. Des. 3 (2006) 2. https://doi.org/10.1007/s10999006-9016-9 
5 Y. Wang, Q. Huang, M. Zhou, and Z. Xu: Arch. Appl. Mech. 77 (2007) 461. https://doi.org/10.1007/s00419006-0106-9

6 R. Steinbuch: J. Bionic. Eng. 8 (2011) 335. https://doi.org/10.1016/S1672-6529(11)60036-X

7 Q. Liu, F. Zhang, N. Hu, H. Wang, K. Hsia, and P. Wang: J. Bionic. Eng. 9 (2012) 494. https://doi.org/10.1016/ S1672-6529(11)60146-7

8 W. Wu, M. Huang, and X. Gu: Rob. Auton. Syst. 100 (2018) 119. https://doi.org/10.1016/j.robot.2017.11.006

9 I. Kovalev: J. Bionic. Eng. 5 (2008) 224. https://doi.org/10.1016/S1672-6529(08)60028-1

10 P. Gao, D. Sun, P. Liang, and Y. Shen: J. Low. Freq. Noise 34 (2014) 119. https://doi.org/10.3969/ j.issn.1006-1335.2014.03.025

11 C. Zhang, D. Mei, and Z. Chen: CHME 17 (2006) 2278. https://doi.org/10.3321/j.issn:1004-132X.2006.21.019

12 M. Lan and P. Wei: Acta Mech. 225 (2014) 1779. https://doi.org/10.1007/s00707-013-0984-1

13 W. Cheng: Dev. Appl. Mater. 27 (2012) 33. http://www.cqvip.com/QK/92793X/201204/1002027457.html

14 M. Zheng, W. Wang, and Z. Chen: China Rubber Ind. 50 (2003) 462. https://doi.org/10.3969/j.issn.1000890X.2003.08.003 